\title{
Téoros
}

Revue de recherche en tourisme

\section{Des besoins partiellement satisfaits}

Sondage sur la recherche en tourisme

\section{Nicole Chenette, Lise Marcil et Gustavo Del Valle}

Volume 1, numéro 2, 2e trimestre 1982

La recherche en tourisme : forces et faiblesses

URI : https://id.erudit.org/iderudit/1080854ar

DOI : https://doi.org/10.7202/1080854ar

Aller au sommaire du numéro

Éditeur(s)

Université du Québec à Montréal

ISSN

0712-8657 (imprimé)

1923-2705 (numérique)

Découvrir la revue

Citer cet article

Chenette, N., Marcil, L. \& Del Valle, G. (1982). Des besoins partiellement satisfaits : sondage sur la recherche en tourisme. Téoros, 1(2), 9-12.

https://doi.org/10.7202/1080854ar d'utilisation que vous pouvez consulter en ligne.

https://apropos.erudit.org/fr/usagers/politique-dutilisation/ 


\title{
Des besoins partiellement satisfaits
}

\author{
Sondage sur la recherche en tourisme \\ Par Nicole Chenette, Lise Marcil et Gustavo Del Valle
}

L'importance croissante de la recherche, dans les sociétés industrielles avancées, comme source de connaissance des phénomènes sociaux et naturels, nous a porté à interroger le domaine touristique. II s'agissait de déterminer, par un sondage, (1) la place de la recherche dans le milieu du tourisme. spécifiquement dans le monde des associations à but non lucratif.

Notre interrogation touchait les organismes associatifs québécois des milieux du loisir et de la culture, ayant une certaine orientation touristique. Ces derniers ont été recensés à partir de différents bottins qui regroupent les organismes concernés.

Pour atteindre notre objectif, nous avons élaboré un questionnaire avec la collaboration du professeur M. Jean Stafford. Le questionnaire comprenait quatre volets: les caractéristiques des associations, les coûts de la recherche, l'utilisation des résultats, et les perspectives de la recherche en tourisme. L'ensemble de la "population" (les associations) à qui fut posté le questionnaire recevait, trois semaines aprés l'envoi, une lettre de rappel. Dans le cas des organismes qui ont leur siège à Montréal, le rappel se fit par téléphone. Sur les cent deux (102) organismes touchés, cinquante-deux (52) nous ont répondu.

Nous avons traité mécanographiquement les données, ainsi recueillies. pour ensuite procéder à des analyses de fréquences. Cette enquête doit être considérée comme préliminaire et, à ce titre, ces données ne doivent pas être perçues comme exhaustives.

\section{Le profil des organismes}

Dans l'ensemble des organismes répondant, $46 \%$ sont à vocation régionale; $48 \%$ opèrent à l'échelle de la province; $68.6 \%$ sont accessibles à toute la population. Selon leur champ d'activité, $42.3 \%$ se classent strictement dans le tourisme.

(1) Le sondage a due effectue du 16 mars au 26 auril 1982

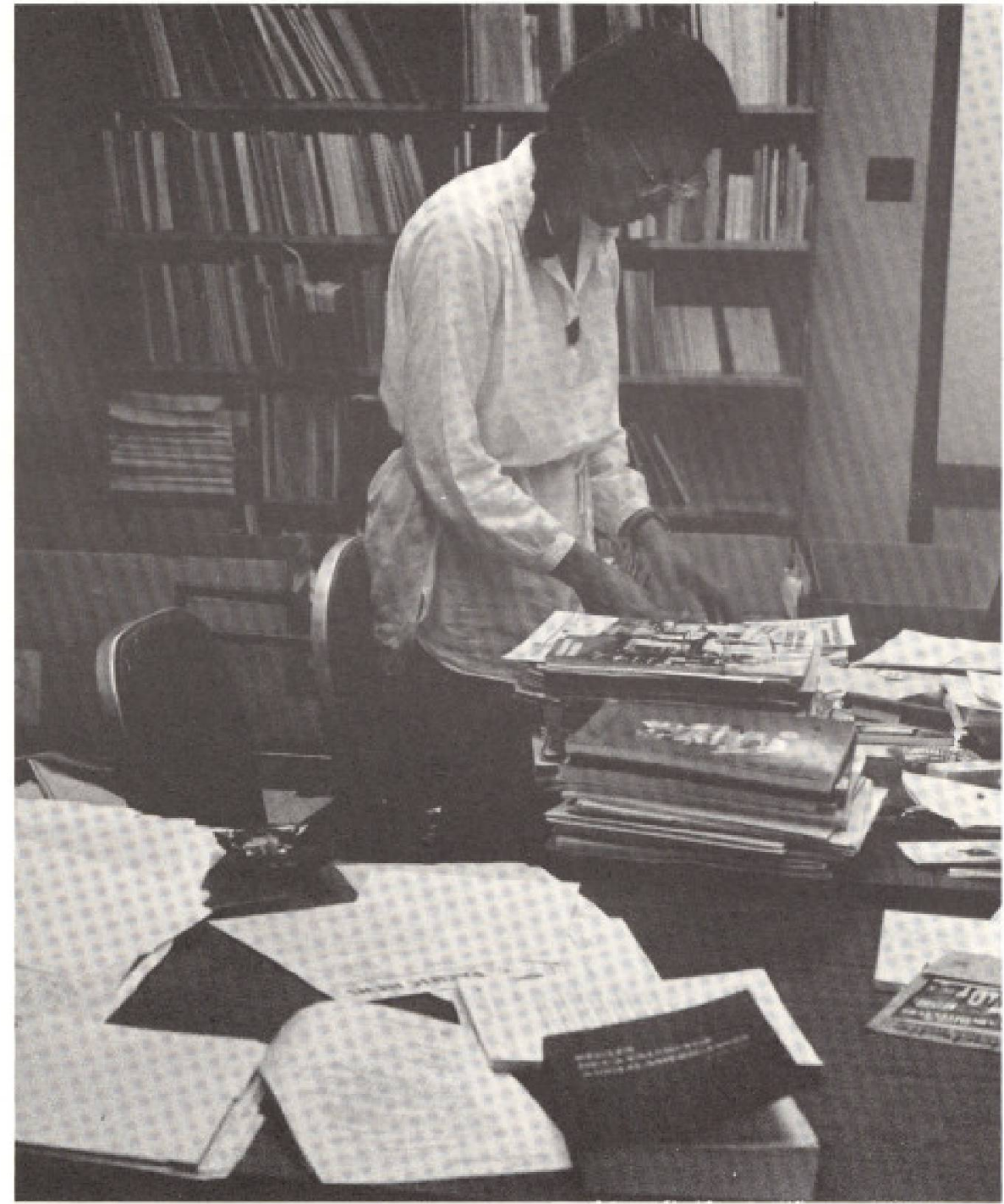

Tableau A

Répartition de la "population" (associations) sur la base de l'expérience de recherche.

$$
\text { Groupe } 1 \text { Groupe } 2 \text { Population }
$$

$\begin{array}{cccc}\text { Nombre } & 37 & 15 & 52 \\ \% & 71.15 \% & 28.85 \% & 100 \%\end{array}$




\section{Tableau B}

Type de recherche [objet]

Clientèle

Promotion

Gestion

Économique

Socio-culturel

Autres

\begin{tabular}{rr} 
Nombre & \multicolumn{1}{c}{$\%$} \\
11 & $14.3 \%$ \\
3 & $3.9 \%$ \\
5 & $6.5 \%$ \\
26 & $33.8 \%$ \\
29 & $37.7 \%$ \\
3 & $3.9 \%$
\end{tabular}

\section{Tableau C}

L'importance et la possibilité de réalisation de certaines recherches pour les organismes qui n'ont pas fait de recherche (groupe 2).

Études de clientèle actuelle
Études de clientèle potentielle
Études de promotion
Études de I'offre
Études des avantages/coûts

Importance

(assez et très)

$86.7 \%$

$73.4 \%$

$86.6 \%$

$40.0 \%$

$40.0 \%$

réalisable
(assez et très)
$53.3 \%$
$53.3 \%$
$46.7 \%$
$46.7 \%$
$46.7 \%$

$46.7 \%$

\section{Tableau D}

L'importance et la possibilité de réalisation de certaines recherches pour les organismes qui ont déjâ fait au moins une recherche (groupe 1).

Etudes de clientèle actuelle
Etudes de clientele potentielle
Etudes de promotion
Etudes de l'offre
Etudes des avantages/coúts

Importance
(assez et très)
$75.7 \%$
$73.0 \%$
$94.6 \%$
$62.1 \%$
$78.3 \%$

\section{réálisable} (assez et très)

$62.1 \%$

$62.1 \%$

$81.0 \%$

$48.6 \%$

Eludes des avantages/couts
L'état de la recherche

L'expérience de recherche

Ces organismes révélent dans leur description un degré considérable de disparité si bien qu'il a été impossible de trouver une base de comparaison intrinsèque, quelle que soit la caractéristique considérée. Alors pour apprécier l'état de la recherche dans ce secteur touristique, nous choisissons l'expérience de recherche comme critère distinctif. Autrement dit, nous regroupons les associations de la manière suivante: celles qui ont au moins une expérience de recherche (groupe 1) et celles qui n'en ont jamais fait (groupe 2). De la, nous étudierons la situation de la recherche.

En polarisant de la sorte les données, il apparait que plus de $70 \%$ des organismes ayant participé au sondage ont au moins une expérience de recherche alors que $28.8 \%$ n'en ont jamais réalisé.

Mais, avant d'étudier en détail les conditions de réalisation des recherches, ne convient-il pas de voir les facteurs qui, du point de vue des caractéristiques des organismes. auraient une incidence sur la pratique de la recherche dans le milieu touristique?

D'une façon générale, les différentes caractéristiques utilisées antérieurement pour décrire les organismes ne paraissent pas influencer substantiellement la réalisation ou la non-réalisation d'une recherche. La seule qui semblerait avoir une incidence sur la recherche, c'est la durée d'existence des organismes. En effet, les organismes de moins de 5 ans s'avèrent être les plus engagés dans la recherche. puisqu'ils composent $35.1 \%$ des organismes qui ont au moins une expérience de recherche.

L'experience de recherche des organismes s'étend sur une période de onze ans mais $62.3 \%$ des recherches ont été réalisées depuis 1980. 37 organismes ont réalisé un total de 77 recherches, réparties de la façon suivante: 13 organismes n'ont entrepris qu'une seule recherche, 7 organismes en ont fait 2 et 17 autres en ont réalisé au moins 3 .

Les objets de la recherche

Sur quoi portent les diverses recherches? Quelle est leur orientation? Nous avons présenté aux associations 6 catégories. 
Dans le tableau $B$, trois catégories retiennent particulièrement notre attention de par l'importance qu'il leur a été accordée: ce sont les recherches à caractère socio-culturel $(37.7 \%)$ les études sur les questions économiques $(33.8 \%)$ et les recherches visant une meilleure connaissance de la çlientèle (14.3\%).

\section{L'organisation de la recherche}

Des multiples aspects qui composent l'organisation de la recherche en général, nous nous sommes attardés sur ceux qui paraissaient les plus pertinents à notre étude: le personnel de la recherche, le coût et le financement, et finalement la diffusion (à laquelle nous avons ajouté l'exploitation des résultats de recherche).

\section{- Personnel}

Les ressources humaines qui ont contribué à la réalisation des différentes recherches proviennent de 3 sphères, soit des organisations ellesmêmes $(62 \%)$, soit des firmes privées (4\%), soit des chercheurs indépendants $(24 \%)$. Parfois les organismes se doublaient de pigistes ou d'un personnel venant de firmes privées. Est-ce à dire que les organismes qui ont entrepris des recherches disposaient de leurs propres chercheurs? La question reste en suspens!

- Coût et financement

En général les coûts de recherches entreprises variaient de $\$ 50.00$ à $\$ 58.000 .00$ mais $38.2 \%$ des recherches couttaient entre $\$ 2,000,00$ et $\$ 10,000.00$. D'autre part $47.4 \%$ des recherches réalisées étaient financées par les organismes tandis que, dans $38.2 \%$ des cas, les fonds provenaient de subventions particulières.

- Diffusion et exploitation des résultats de recherche

$67.1 \%$ des recherches menées ont eu une diffusion externe et interne. Les recherches n'ont pas été vaines puisque les responsables les déclarent utiles a l'organisme à $92.1 \%$. Comment expliquer alors que ces organismes n'en font pas plus sills conviennent. dans une si forte proportion, de l'utilité des résultats?

\section{Tableau E}

Le souhait et la faisabilité des recherches réalisées conjointement pour les organismes qui n'ont pas fait de recherche (groupe 2).

$\begin{array}{cc}\begin{array}{c}\text { Souhait } \\ \text { (assez et très) }\end{array} & \text { faisable } \\ \text { (assez et très) } \\ 66.7 \% & 73.4 \% \\ 66.7 \% & 66.7 \% \\ 66.7 \% & 53.4 \% \\ 73.3 \% & 46.7 \% \\ 60.0 \% & 46.7 \%\end{array}$

\section{Tableau F}

Le souhait et la faisabilité des recherches réalisées conjointement pour les organismes qui ont fait au moins une recherche (groupe 1).

$\begin{array}{lcc}\text { Recherches conjointes } & \begin{array}{c}\text { Souhait } \\ \text { (assez et très) }\end{array} & \begin{array}{c}\text { faisable } \\ \text { (assez et très) }\end{array} \\ \text { Méthodologie commune } & 91.9 \% & 75.6 \% \\ \text { Partage des ressources humaines } & 82.2 \% & 72.9 \% \\ \text { Base de données dans un centre } & 86.5 \% & 64.9 \% \\ \text { Système statistique unifié } & 91.8 \% & 64.8 \%\end{array}$

Les besoins de recherche dans le milieu touristique

En regard du tableau $C$, relatif aux organismes qui n'ont jamais réalisé de recherche, nous notons que leurs besoins de recherche sont très grands. Pour ce groupe d'organismes, les deux premières priorités ( $86 \%$, chacune) sont les études de clientèle actuelle et les études de promotion; les études de clientèle potentielle obtiennent $73.4 \%$ Les études à caractère principalement économique (études de l'offre et des avantages/coûts) semblent moins importantes que les précédentes, $(40 \%$, pour chacune de ces catégories). Par contre, nous constatons une nette différence entre les études qui s'avèrent importantes et leur possibilité de réalisation.

Dans le tableau D, ce qui paraît primordial pour les organismes qui ont réalisé au moins une recherche, c'est d'abord et surtout des études de promotion (94.6\%), ensuite des études des avantages/coúts $(78.3 \%)$ et en troisième lieu, des études de clientèle qui recueillent au moins $73 \%$. Comparativement aux études mentionnées, celles de l'offre ont moins d'importance $(62.1 \%)$. Si nous considérons les possibilités de réalisation de ces études, la même tendance se maintient.
En superposant les deux tableaux, I'étude de promotion semble prioritaire pour les 2 groupes. Pour les études portant sur l'offre, aucun des 2 groupes n'a fait montre de beaucoup d'intérêt. Nous faisons remarquer la persistance d'une idée communément admise, l'inadéquation entre les besoins et leur réalisation.

II apparaît à ce niveau un besoin de recherche nettement prononcé, car tant du groupe 1 que du groupe 2. la tendance est à la hausse pour élaborer et réaliser des recherches. Même si $70 \%$ des organismes interrogés ont réalisé au moins une recherche, le besoin de recherche n'est pas pour autant comblé. 
L'avenir de la recherche

L'utilisation des résultats de recherches

II apparaît que les résultats de recherches futures serviront, principalement, à des fins de planification, option qui a été considérée comme importante à $91.9 \%$ pour le groupe 1 et $93.3 \%$ pour le groupe 2 ; en deuxième et troisième place viennent respectivement la gestion et l'animation. II est intéressant aussi de noter la variation de l'importance accordée à la diffusion externe entre le groupe $1(75.6 \%)$ et le groupe 2 (40\%).

\section{Les recherches conjointes}

En ce qui concerne la réalisation des recherches conjointes au tableau $\mathrm{E}_{\text {, }}$ pour le groupe 2 elle n'est pas seulement souhaitable $(66.7 \%)$ mais ces organismes considèrent la faisabilité de ces recherches dans une large proportion $(73.4 \%)$. Un autre souhait qui parait élevé, c'est la possibilité de posséder une base commune de données $(73.3 \%)$. L intérêt n'apparaît pas aussi marqué pour une méthodologie commune (66\%), pour un partage des ressources humaines $(66 \%)$ et pour un systeme statistique standard $(60 \%)$. De plus, ce groupe d'organismes paraît moins enclin à favoriser la concrétisation de ces derniers critères.

Au tableau $F$, le groupe 1 semble par contre, très ouvert à toutes les formes de mise en commun, ceci autant en terme de souhait qu'en terme de faisabilité. Les recherches conjointes $(91.9 \%)$, une base commune de données dans un centre (91.8\%) et une méthodologie commune (89.2\%) sont nettement désirées et seul le système statistique unifié semble ètre moins souhaitable (78.3\%). Si pour le groupe 1 les souhaits sont très forts, lorsque l'on considere les possibilites de concrétisation. nous constatons une nette différence. Les recherches conjointes et une méthodologie commune semblent les plus probables.

D'une façon générale, le groupe 1 est beaucoup plus disponible à la mise en commun que le groupe 2 . Une vision d'ensemble des deux tableaux fait apparaitre la nette prédisposition de ces organismes, non seulement à avoir mais aussi à realiser des recherches conjointes et une méthodologie commune. Pourtant, au regard d'un partage des ressources humaines, d'une même base de données et d'une standardisation des statistiques, nous ne pouvons que reconnaître que si le souhait est présent, la réalisation semble être plus problématique.
Le budget prévisionnel alloué aux recherches

Le taux élevé de non-réponses en regard des prévisions budgétaires pour les recherches singularise cette question. Particulièrement pour le groupe 2, le taux de non-réponses croît avec les années soit $66.7 \%, 86.7 \%$ et $86.7 \%$ pour la période allant de 1983 a 1985. Aucun organisme du groupe 2 n'a pu faire une prevision de plus de $\$ 8,000.00$ pour chacune des trois prochaines années. Le taux de nonréponses est aussi élevé pour le groupe 1 , il passe de $54.1 \%$ à $62.2 \%$ et à $70.3 \%$ pour la même période, de 1983 à 1985.

Nous pouvons dire que l'índécision ou l'impossibilité de prévoir un budget de recherche caractérisent I'ensemble des organismes.

\section{Conclusion}

Bien que notre définition englobe des organismes disparates sur le plan des caractéristiques, il nous a été tout de même possible de les réunir en deux groupes pertinents à notre problématique. Ceci nous a permis de connaitre la situation de la recherche dans le milieu touristique, du moins les associations sans but Iucratif, d'identifier un besoin de recherche et de dégager quelques avenues possibles.

En effet, qu'il s'agisse d'organismes ayant une expérience de recherche ou non, le besoin de recherche est manifeste pour la plupart des aspects considérés. Or, plus de $70 \%$ des organismes ont déjà entrepris des recherches; seraient-ce des contraintes de ressources humaines ou de budget qui les ont empeché d'investir davantage dans ce domaine?

Malgré le recours à des chercheurs internes ou à des pigistes, le groupe 1 n'arrive pas à combler ses besoins de recherche. Nous pouvons très bien nous imaginer qu'un tel besoin est encore plus crucial pour le groupe 2. En considérant les sommes d'argent allouées à la recherche, on peut se demander s'il n'y a pas une insuffisance sur ce plan et si celle-ci n"expliquerait pas, par ailleurs, le fait que ces organismes n'ont pu couvrir au moins une partie de leurs besoins.
Certes, nous ne pouvons soutenir I'hypothèse que les contraintes budgétaires sont plus grande chez le groupe 2 que chez le groupe 1. Mais, face aux difficultés des organismes, pour né pas dire leur incapacité à prévoir un budget de recherche, et au fait que $70 \%$ des organismes disposaient chacun d'un budget annuel ne dépassant pas $\$ 200,000.00$, nous pouvons nous demander (si leur survie est annuelle) comment, dans ces conditions, les organismes pourraient penser à une planification ou à des recherches?...

Dans cet ensemble de besoins. quelques concensus se dégagent. Les organismes interrogés sont ouverts à réaliser des recherches conjointes sur une mème base méthodologique et ils optent nettement pour des études orientées vers la planification.

Cependant, les contradictions sont énormes et portent sur des éléments clés pour l'avenir de la recherche, hypothequant de ce fait le niveau consensuel. Comment peut-on. premièrement réaliser des recherches conjointes si on ne s'entend pas sur une mise en commun de ressources humaines, lorsque, par ailleurs, les organismes qui ont entrepris des recherches ont fait appel à des pigistes ou à des firmes privées? Comment peuton, deuxièmement, réaliser des recherches conjointes lorsque la standardisation des statistiques n'obtient pas l'accord des organismes impliqués et qu'une base de données dans un même centre apparait selon eux, peu réalisable?

Face à un besoin aussi important de recherche, la possibilité de concertation - qui pourrait être une issue - semble bien minime!

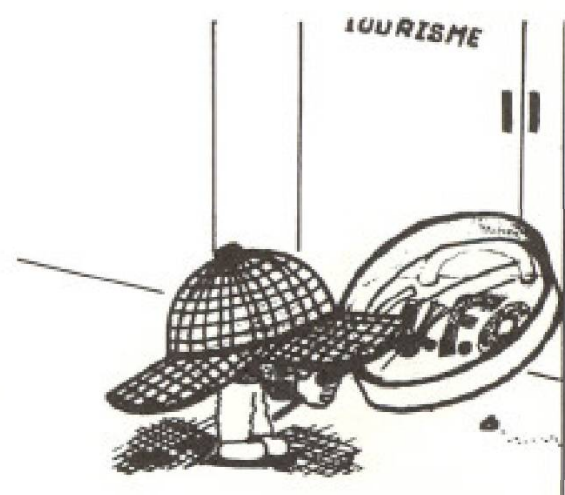

tre 